\title{
Two-dimensional numerical study for relativistic outflow from strongly magnetized neutron stars
}

\author{
Jin Matsumoto ${ }^{1,2}$, Youhei Masada ${ }^{3}$, Eiji Asano ${ }^{1}$ \\ and Kazunari Shibata ${ }^{1}$ \\ ${ }^{1}$ Kwasan and Hida Observatories, Kyoto University, Kyoto, Japan \\ email: jin@kusastro.kyoto-u.ac.jp \\ ${ }^{2}$ Department of Astronomy, Kyoto University, Kyoto, Japan. \\ ${ }^{3}$ Institute of Astronomy and Astrophysics, Academia Sinica, Taiwan, R.O.C.
}

\begin{abstract}
Using special relativistic magnetohydrodynamic simulation, the nonlinear dynamics of the magnetized outflow triggered on the magnetar surface is investigated. It is found that the strong shock propagates in the circumstellar medium in association with the expanding outflow. The shock velocity $v_{\text {sh }}$ depends on the strength of the dipole field anchored to the stellar surface $B_{\text {dipole }}$ and is described by a simple scaling relation $v_{\mathrm{sh}} \propto B_{\text {dipole }}{ }^{0.5}$. In addition, the outflow-driven shock can be accelerated self-similarly to the relativistic velocity when the density profile of the circumstellar medium is steeper than the critical density profile, that is $\alpha \equiv \mathrm{d} \log \rho(\mathrm{r}) / \mathrm{d} \log \mathrm{r} \lesssim \alpha_{\text {crit }}=-5.0$, where the density is set as a power law distribution with an index $\alpha$ and $r$ is the cylindrical radius. Our results suggest that the relativistic outflow would be driven by the flaring activity in a circumstellar medium with a steep density profile.
\end{abstract}

Keywords. Relativity - MHD - stars: neutron - methods: numerical

\section{Introduction}

Explosive magnetic energy release is widely accepted to play a crucial role in the flaring activity observed on ultra-strongly magnetized neutron stars, so called "magnetars" (B $\gtrsim 10^{14} \mathrm{G}$; Thompson \& Duncan 1995). The physical mechanism of the energy release on the magnetar surface and the characteristics of the magnetically-driven outflow are not fully understood. Here we investigate, using axisymmetric special relativistic magnetohydrodynamic simulation, the nonlinear dynamics of the magnetized outflow triggered by the magnetic explosion on the magnetar surface.

As an initial setting, we set the hydrostatic circumstellar medium to have a density $\rho(r) \propto r^{\alpha}$ and a dipole magnetic field, $B_{r}, B_{\theta} \propto B_{0} r^{-3}$, where $\alpha$ is the power-law index, $B_{0}$ is the surface field strength, and $r$ is the cylindrical radius. To initiate the expanding outflow, an azimuthal shearing motion is added around the equatorial surface, and which produces the azimuthal component of the magnetic field (Mikic \& Linker 1994).

\section{Results}

Figure 1 shows the density contour and the magnetic field lines in the meridional plane for the case where $\alpha=3.44$ and $B_{0}=10^{14} G$. The twisted magnetic field created around the equatorial surface, by the shearing motion, drives the supersonically expanding outflow. It produces a strong shock wave propagating through the circumstellar medium. The relation between the strength of the initial dipole field and the shock velocity is 

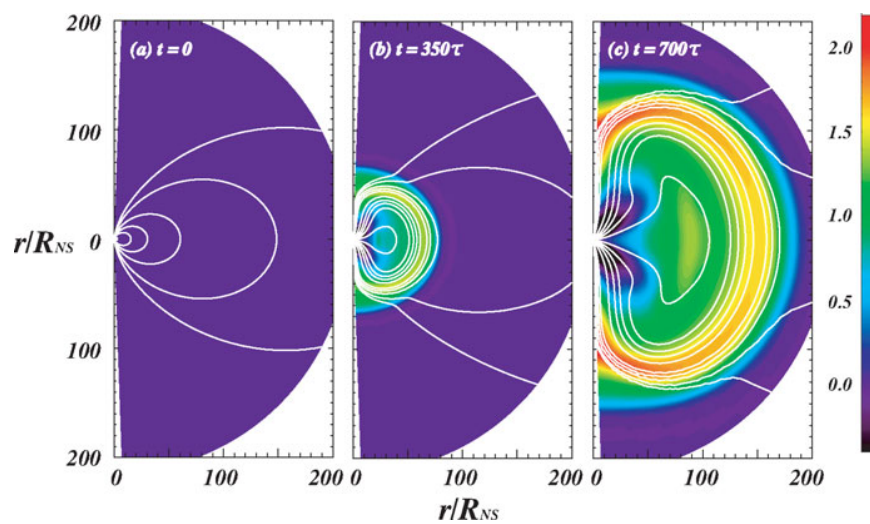

Figure 1. The time evolution of the density distribution (log scale) and magnetic field lines. The left, middle and right panels are corresponding to those in $t / \tau=0,350$, and 700 respectively, where $\tau=R_{\mathrm{NS}} / c \sim 4.5 \times 10^{-5}$ sec is the dynamical timescale.
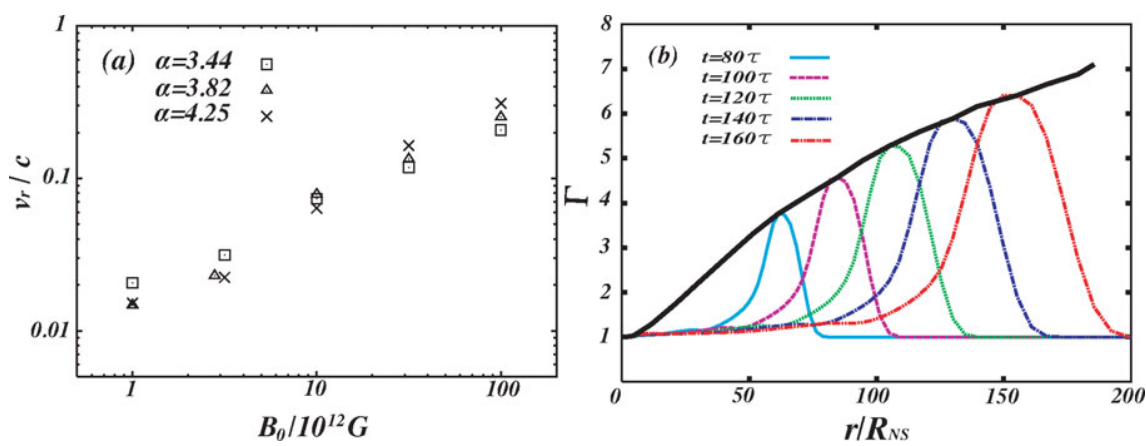

Figure 2. Panel (a): The relation between the strength of the dipole magnetic field and the shock velocity. Panel (b): The time evolution of the Lorentz factor in the case with $\alpha=-7.29$. A black solid line represents the Lorentz factor of the plasma measured in the comoving frame of the shock surface.

examined by varying $B_{0}$ from $10^{12}$ to $10^{14} \mathrm{G}$, and which is shown in Figure 2a. We can find from this figure, the scaling relation $v_{\text {sh }} \propto B_{\text {init }}{ }^{0.5}$ when $\alpha=3.44,3.82$, and 4.25.

The physical properties of the shock wave can be changed due to the initial density profile of the circumstellar medium (i.e., index $\alpha$ ). It is accelerated rapidly to the relativistic velocity when $\alpha$ is smaller than $\approx-5$. Note that a smaller index $\alpha$ corresponds to a steeper density profile. The relativistic shock wave can thus be accelerated through the circumstellar medium only with a steep density profile. Figure $2 \mathrm{~b}$ illustrates the time evolution of the shock velocity in the relativistic expansion case with $\alpha=-7.29$. The vertical axis represents the Lorentz factor of the plasma. The horizontal axis is the cylindrical radius. The curves indicate the cases where $t / \tau=80,100,120,140$, and 160 respectively. It can be seen from this figure that the outflow must be accelerated selfsimilarly to the relativistic velocity. Our nonlinear study suggests that the expanding outflow is accelerated in association with the flaring activity on the magnetar surface.

\section{References}

Mikic, Z. \& Linker, J. A. 1994, ApJ 430, 898

Thompson, C. \& Duncan, R. C. 1995, MNRAS 275, 255 